\title{
Individual, Social, and Environmental Correlates of Active Transportation Patterns in French Women
}

\author{
Camille Perchoux, ${ }^{1,2}$ Christophe Enaux, ${ }^{3}$ Jean-Michel Oppert, ${ }^{4,5}$ Mehdi Menai, ${ }^{4}$ \\ Hélène Charreire, ${ }^{4,6}$ Paul Salze, ${ }^{3}$ Christiane Weber, ${ }^{3}$ Serge Hercberg, ${ }^{4,7}$ Thierry Feuillet, ${ }^{4,8}$ \\ Franck Hess, ${ }^{3}$ Célina Roda, ${ }^{4}$ Chantal Simon, ${ }^{1}$ and Julie-Anne Nazare ${ }^{1}$ \\ ${ }^{1}$ CRNH-Rhône-Alpes, CARMEN, INSERM U1060, Université de Lyon 1, INRA U1235, Lyon, France \\ ${ }^{2}$ Luxembourg Institute of Socio-Economic Research (LISER), Esch-sur-Alzette, Luxembourg \\ ${ }^{3}$ Laboratoire, Image, Ville et Environnement, Université de Strasbourg, Strasbourg, France \\ ${ }^{4}$ Equipe de Recherche en Epidémiologie Nutritionnelle (EREN), Centre de Recherche en Epidémiologie et Biostatistiques, \\ INSERM U1153, INRA U1125, Cnam, COMUE Sorbonne Paris Cité, Université Paris 13, Bobigny, France \\ ${ }^{5}$ Department of Nutrition, Pitié-Salpêtrière Hospital (AP-HP), Institute of Cardiometabolism and Nutrition (ICAN), \\ Université Pierre et Marie Curie-Paris 6, Paris, France \\ ${ }^{6}$ Department of Geography, Lab-Urba, Université Paris-Est, Créteil, France \\ ${ }^{7}$ Department of Public Health, Hôpital Avicenne (AP-HP), Bobigny, France \\ ${ }^{8}$ Department of Geography, UMR 7533, LADYSS, Université Paris 8, Saint-Denis, France
}

Correspondence should be addressed to Julie-Anne Nazare; julie-anne.nazare@univ-lyon1.fr

Received 20 January 2017; Revised 4 May 2017; Accepted 23 May 2017; Published 22 June 2017

Academic Editor: Jung-Min Lee

Copyright ( 2017 Camille Perchoux et al. This is an open access article distributed under the Creative Commons Attribution License, which permits unrestricted use, distribution, and reproduction in any medium, provided the original work is properly cited.

\begin{abstract}
The objectives were (1) to define physical activity (PA) and sedentary behaviors (SB) patterns in daily life contexts (work, leisure, and transportation) in French working women from NutriNet-Santé web-cohort and (2) to identify pattern(s) of active transportation and their individual, social, and environmental correlates. 23,432 participants completed two questionnaires to evaluate PA and SB in daily life contexts and individual representations of residential neighborhood and transportation modes. Hierarchical cluster analysis was performed which identified 6 distinct movement behavior patterns: (i) active occupation, high sedentary leisure, (ii) sedentary occupation, low leisure, (iii) sedentary transportation, (iv) sedentary occupation and leisure, (v) active transportation, and (vi) active leisure. Multinomial logistic regressions were performed to identify correlates of the "active transportation" cluster. The perceived environmental characteristics positively associated with "active transportation" included "high availability of destinations around home," "presence of bicycle paths," and "low traffic." A "positive image of walking/cycling," the "individual feeling of being physically active," and a "high use of active transport modes by relatives/friends" were positively related to "active transportation," identified as a unique pattern regarding individual and environmental correlates. Identification of PA and SB context-specific patterns will help to understand movement behaviors' complexity and to design interventions to promote active transportation in specific subgroups.
\end{abstract}

\section{Introduction}

Physical inactivity is recognized as one of the main modifiable lifestyle risk factors for noncommunicable diseases such as cardiovascular disease, diabetes, and certain cancers [1, 2]. According to a recent worldwide report, about $23 \%$ of the European population does not meet WHO targets for sufficient physical activity (PA) for health [3]. Moreover, high levels of sedentary behavior such as prolonged sitting time have been associated with a deleterious health profile and mortality [4]. As demonstrated in a meta-analysis including more than 1 million individuals, jointly examining PA and sedentary behavior (SB) is of the upmost importance as high levels of PA may attenuate the detrimental health impact 
of prolonged sitting time [5]. This also demonstrates the ongoing need for an integrated approach to movementrelated behaviors including both $\mathrm{PA}$ and SB.

Promoting active transportation (walking or cycling) appears as a relevant lever to increase usual PA at population level for several reasons: (1) active transportation has been associated with a higher level of daily total PA in crosssectional and longitudinal studies [6-8]; (2) active transportation is an affordable, convenient, simple, and nonpolluting activity that can easily be integrated into everydaylife routines. However, designing effective PA interventions requires prior knowledge of the correlates (individual, environmental, and social) of usual PA [9].

Based on socioecological models of health behaviors, a large body of research has investigated the individual and environmental factors related to active transportation including individual-level factors such as age, body mass index (BMI), education level, self-efficacy, perceived benefits and barriers, employment status, and environmental-level factors such as street connectivity, walkability and cyclability, density of destination, traffic, population density, and social support [10-15]. Pioneering work in the early 2000s has put forward the need for context-specific analyses of the correlates of PA, more particularly for active transport.

During the course of a 24-hour day, movement-related behaviors alternate over time and PA (low-intensity, moderate to vigorous) and SB "compete" with each other depending on the contexts in which they are undertaken [10]. Therefore, analysis of the context-specific components of movementrelated behavior, such as active transport, should not be performed in isolation but within the complex patterning of the various components of movement-related behaviors [5]. In this perspective, cluster analysis is gaining growing interest in health promotion research by providing valuable information on the way context-specific behaviors are corelated and organized relatively to each other in order to group individuals that present similar lifestyle patterns. Yet only few studies have investigated the patterns of movementrelated behaviors in everyday-life contexts, using cluster or latent class analysis [16-20]. Such approaches are needed to identify the levers to be favored to promote active travel and developing more efficient tailored prevention programs.

Our objectives were (1) to define PA and SBs patterns in daily life contexts (work, leisure, and transportation) in a large sample of French adult women from an ongoing web-cohort, (2) to identify pattern(s) of active transportation, and to (3) to investigate, through a socioecological approach, the individual, social, and environmental correlates of active transportation within comprehensive movement-related behaviors patterns.

\section{Subjects and Methods}

2.1. Population. This study is part of the ACTI-Cités project [21] based on the Nutrinet-Santé Cohort Study, a webbased cohort launched in France in 2009 to evaluate the relationships between nutrition and health [22]. In the ACTICités study, participants of the Nutrinet-Santé Cohort Study aged 18 years or older were invited to complete two web-based questionnaires, as previously reported [10, 23]. The Sedentary and Transport Activity Questionnaire (STAQ) [24] assessed the last 4-week PA and SBs in daily life contexts including work, leisure, and transportation. The questionnaire of the daily life environment (QEVIC) [25] evaluated the individual's representations of their residential neighborhood and transportation modes. This study was conducted according to the guidelines laid down in the Declaration of Helsinki, and all procedures were approved by the Institutional Review Board of the French Institute for Health and Medical Research (IRB Inserm number 0000388FWA00005831) and the Commission Nationale Informatique et Libertés (CNIL number 908450 and no 909216). All participants gave their written electronic informed consent to take part in the study. The present analysis is based on the women subsample of the ACTI-Cités study (71.3\% women).

2.2. Variables of Physical Activity and Sedentary Behaviors. Five variables from the STAQ were computed to evaluate the PA and SB in everyday-life contexts [24].

2.2.1. Physical Activity. Participants were asked to report the past-month frequency, duration, and modes of transportation in different contexts including commuting and utilitarian purposes. The time spent active during transportation (hours/week) was calculated by summing the time per week spent walking, cycling, or using another active transportation mode (i.e., skateboard, etc.). Participants were also asked to report the past-month frequency and duration of 38 types of sport and other active leisure activities. The active time spent during leisure (hours/week) was calculated by summing the time spent per week doing these 38 activities. Participants were also asked to evaluate whether they perceived themselves as physically active individuals (yes/no).

2.2.2. Sedentary Behaviors. Participants were asked to report the average time per workday and nonworkday they spent watching TV, using a computer or a tablet computer or playing videogames, reading, writing, knitting, or other sedentary leisure activities, during the past month. The sedentary time spent during leisure (hours/week) was calculated by summing the time per week spent in each reported sedentary leisure activity. Similarly to the computation of active transportation time, the time spent sedentary during transportation (hours/week) was calculated by summing the time spent in an individual motorized transportation mode (i.e., car, motorbike) and/or using public transports. The time spent sedentary at work (hours/week) was assessed by the mean length of time per workday spent sitting at work, during the past month.

\subsection{Environmental, Social and Individual Variables}

2.3.1. Perceived Residential Environment Attributes. Information regarding the perceived residential environment was obtained using the QEVIC [25]. We considered the following variables: availability of target destinations, ease of walking on sidewalks, presence of bicycle paths, pollution levels of the neighborhood, cleanliness/maintenance of the 
neighborhood, presence of trees, presence of greenspace, neighborhood aesthetics, traffic, and criminality.

2.3.2. Individual Representation of Transportation Modes. The individual's representations of each transportation mode including walking, cycling, individual motorized transportation modes, and public transport were assessed from the QEVIC [25].

2.3.3. Use of Active Transportation Modes by Relatives and Friends. Family's and friends' use of active transportation modes was assessed from the QEVIC [25].

2.3.4. Physical Activity Value by the Family. Participants were also asked in the STAQ whether their family valued PA and more specifically sports.

Detailed information on items of the QEVIC and STAQ questionnaires, variables' computation, and Cronbach's alpha are provided in Supplementary Table 1 in Supplementary Material available online at https://doi.org/10.1155/2017/ 9069730 .

2.4. Covariates. Several sociodemographic variables were considered for adjustment: age, individual education level, having a child under the age of 13 within the household, and living in an urban or a rural setting. Age was used as a continuous variable. Individual education was divided into four classes (no education or primary education, secondary education, lower tertiary education, and upper tertiary education).

2.5. Statistical Analyses. First, we conducted a hierarchical cluster analysis using Ward's method to define a typology of patterns of movement. This method has been used previously in analyses on habitual PA (see $[16,17])$. The cluster analysis accounted for 5 variables of PA and SB in context, including time spent sedentary at work (hours/week), time spent sedentary during leisure activities (hours/week), time spent active during leisure (hours/week), time spent sedentary during transportation (hours/week), and time spent active during transportation (hours/week). We tested solutions between 4 and 8 clusters. Based on the dendrogram and the distribution of the subjects between clusters, we chose a 6-cluster solution representing contrasted patterns of movement with over half of the variation in the five SBs and PA variables being accounted for $\left(R^{2}=0.51\right)$.

Second, we used multinomial logistic regression analysis to identify the correlates of the 6 patterns of movement, using the cluster with the highest level of active transportation as reference (cluster 5). Odds ratios (OR) and 99\% confidence interval (CI) were computed. All variables related to the representation of residential environment, transportation modes, use of active transportation modes by friends and family, and representation of PA were tested for multicollinearity using variance inflation factor [26]. No multicollinearity between variables was detected (all VIF $<2$ ). All variables were first tested one-by-one in unadjusted models. We then combined into one model the variables that were independently associated with at least one cluster controlled for age, individual education, having a child under the age of 13 in the household, and living in an urban versus a rural setting. Finally, we only retained variables that were associated with at least one cluster in the multivariate model.

Sensitivity analysis was performed to evaluate the stability of the cluster analysis. Hierarchical cluster analysis was replicated on a random $10 \%$ of the initial sample to evaluate whether participants similarly aggregated in subsamples [17]. All analyses were performed with SAS 9.4 (SAS Inst., Cary, NC, USA).

\section{Results}

3.1. Characteristics of the Study Population. Among 38,913 women who filled in the STAQ and the QEVIC in 2013, 619 women were excluded for self-reported motor impairments $(N=619)$ and/or for self-reported limitations on walking $100 \mathrm{~m}(N=562)$ and/or for reporting implausible PA or sedentary activity values (i.e., extreme reported values above the 99.5 percentile for each PA and SB activity) $(N=1630)$. Additionally, we excluded 12,864 participants who did not report a work or study activity, or who were retired. The final sample included 23,432 participants. Descriptive information on the sample (demographics, context-specific PA and SB) is provided in Table 1.

\subsection{Clusters of Physical Activity and Sedentary Behaviors by} Daily Life Contexts. Descriptive statistics for the six identified clusters including both active and sedentary behaviors are presented in Table 2. Cluster 1 (30.3\% of the population) was mainly characterized by the lowest duration of SB at work. Individuals in this cluster also accumulated high relative levels of leisure time. This cluster was labeled "active occupation, high sedentary leisure." Cluster 2 (50.8\% of the population) mainly captured highly sedentary individuals at work. Individuals in this cluster also accumulated low absolute and relative levels of leisure time. This cluster was labeled "sedentary occupation, low leisure." Cluster 3 (3.3\% of the population) had the highest duration of sedentary transportation and the second highest duration for sedentary leisure time activities. This cluster was labeled "sedentary transportation." Cluster 4 (8.0\% of the population) had the highest duration of sedentary leisure activities and the second highest duration of SB at work. This cluster was labeled "sedentary occupation and leisure." Cluster 5 (5.5\% of the population) had the highest duration of active transportation and the second highest duration in active leisure. This cluster was labeled "active transportation." Cluster 6 (2.1\% of the population) had the highest duration in active leisure. Participants in this cluster also accumulated the second highest duration in sedentary leisure. This cluster was labeled "active leisure."

Sensitivity analysis based on a random sample representing $10 \%$ of the initial sample showed similar patterns of movement as the whole sample, with a relatively strong agreement (Cramer's $V=0.69$ ). Characteristics of study participants by clusters are presented in Table 3. 
TABLE 1: Characteristics of the study population ( $N=23,432$ women).

\begin{tabular}{|c|c|c|}
\hline Variables & $\%$ or mean & SD \\
\hline \multicolumn{3}{|l|}{ Sociodemographics } \\
\hline Age (mean, years) & 42.6 & $(11.5)$ \\
\hline Living with a partner (\%) & 69.9 & - \\
\hline Living with a child under the age of $13(\%)$ & 30.9 & - \\
\hline \multicolumn{3}{|l|}{ Individual education level (\%) } \\
\hline High & 38.8 & - \\
\hline Middle-high & 34.0 & - \\
\hline Middle-low & 17.9 & - \\
\hline Low & 9.4 & - \\
\hline \multicolumn{3}{|l|}{ Professional status (\%) } \\
\hline Farmer & 0.4 & - \\
\hline Craftsperson, company head & 1.8 & - \\
\hline Executive (liberal profession, engineer) & 32.1 & - \\
\hline Intermediate profession (technician, teacher, and supervisor) & 28.1 & - \\
\hline Employee & 30.0 & - \\
\hline Laborer worker & 1.4 & - \\
\hline Other & 6.2 & - \\
\hline Living in a urban setting (\%) & 89.7 & - \\
\hline \multicolumn{3}{|l|}{ Physical activity and sedentary behavior by context } \\
\hline \multicolumn{3}{|l|}{ Leisure } \\
\hline Leisure time spent active (h/week) & 2.7 & $(3.9)$ \\
\hline Leisure time spent sedentary (h/week) & 33.8 & $(21.9)$ \\
\hline Total leisure time (h/week) & 36.5 & $(22.2)$ \\
\hline \multicolumn{3}{|l|}{ Transportation } \\
\hline Time spent walking/cycling for transportation (h/week) & 2.2 & $(4.4)$ \\
\hline Time spent sedentary for transportation (h/week) & 2.0 & $(3.5)$ \\
\hline Total transportation time (h/week) & 4.2 & $(5.9)$ \\
\hline \multicolumn{3}{|l|}{ Work } \\
\hline Time spent sedentary at work (h/week) & 21.9 & $(15.1)$ \\
\hline Total active time (h/week) & 5.1 & $(6.2)$ \\
\hline Total sedentary time (h/week) & 57.5 & $(28.4)$ \\
\hline
\end{tabular}

3.3. Association between Patterns of Movement Behaviors and Perceived Environmental Characteristics, Representation of Transportation Modes, and Physical Activity. Cluster 5 "active transportation" was used as reference since it represented the highest level of active transport, as well as the highest percentage of individuals who reported active transportation. Unadjusted associations between clusters and perceived environmental characteristics, representation of transportation modes, friends and relatives' use of active transportation modes, and PA (not adjusted for each other) are provided in Supplementary Table 2. Multivariate associations are presented in Table 4.

3.3.1. Cluster 1 "Active Occupation, High Sedentary Leisure" versus Cluster 5 "Active Transportation". Among the perceived environmental characteristics, a high availability of destinations and a high presence of bicycle paths strongly decreased the odds of belonging to Cluster 1 "active occupation, high sedentary leisure" compared with the referent Cluster 5. A perceived highly polluted and low aesthetic residential neighborhood decreased the odds of belonging to Cluster 1. A high positive representation of walking and biking and inversely a low positive representation of individual motorized transportation modes and representing oneself as an active person decreased the odds of belonging to Cluster 1. As for social environment, the likelihood of belonging to Cluster 1 was negatively associated with a high use of walking and cycling among relatives.

3.3.2. Cluster 2 "Sedentary Occupation, Low Leisure" versus Cluster 5 "Active Transportation". Cluster 2 "sedentary occupation, low leisure" presented the same associations with the perception of residential environment and social environment, as Cluster 1 compared with Cluster 5. Moreover, as for 


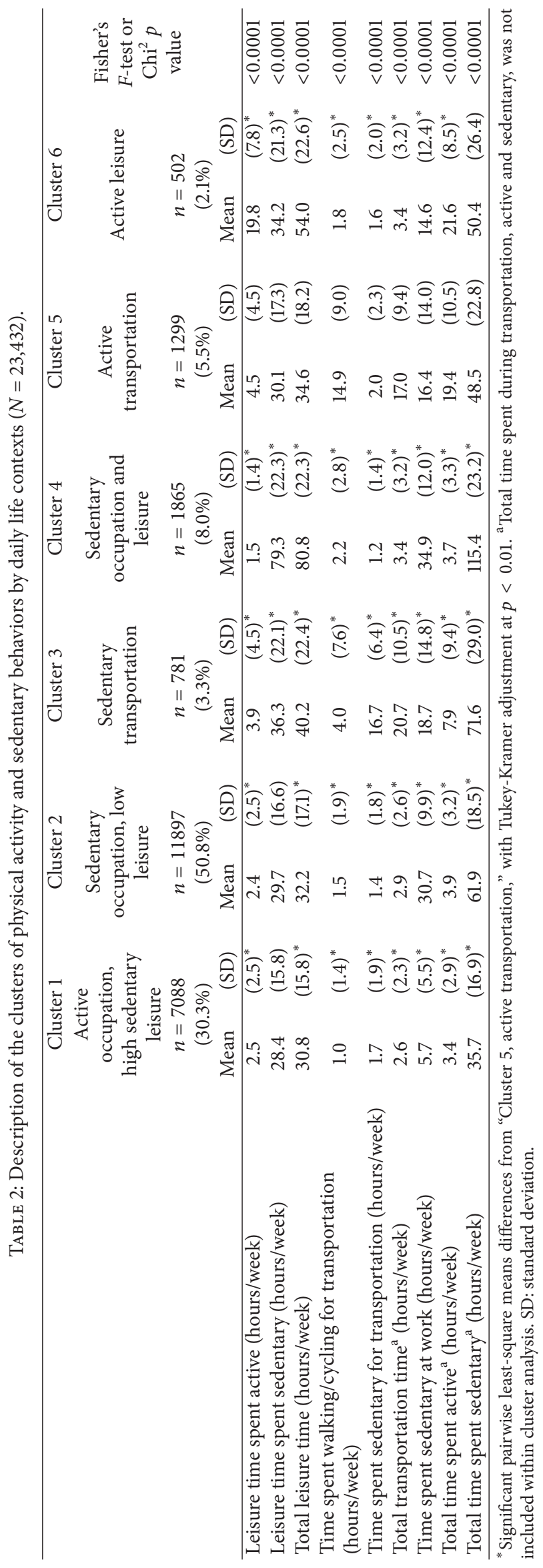




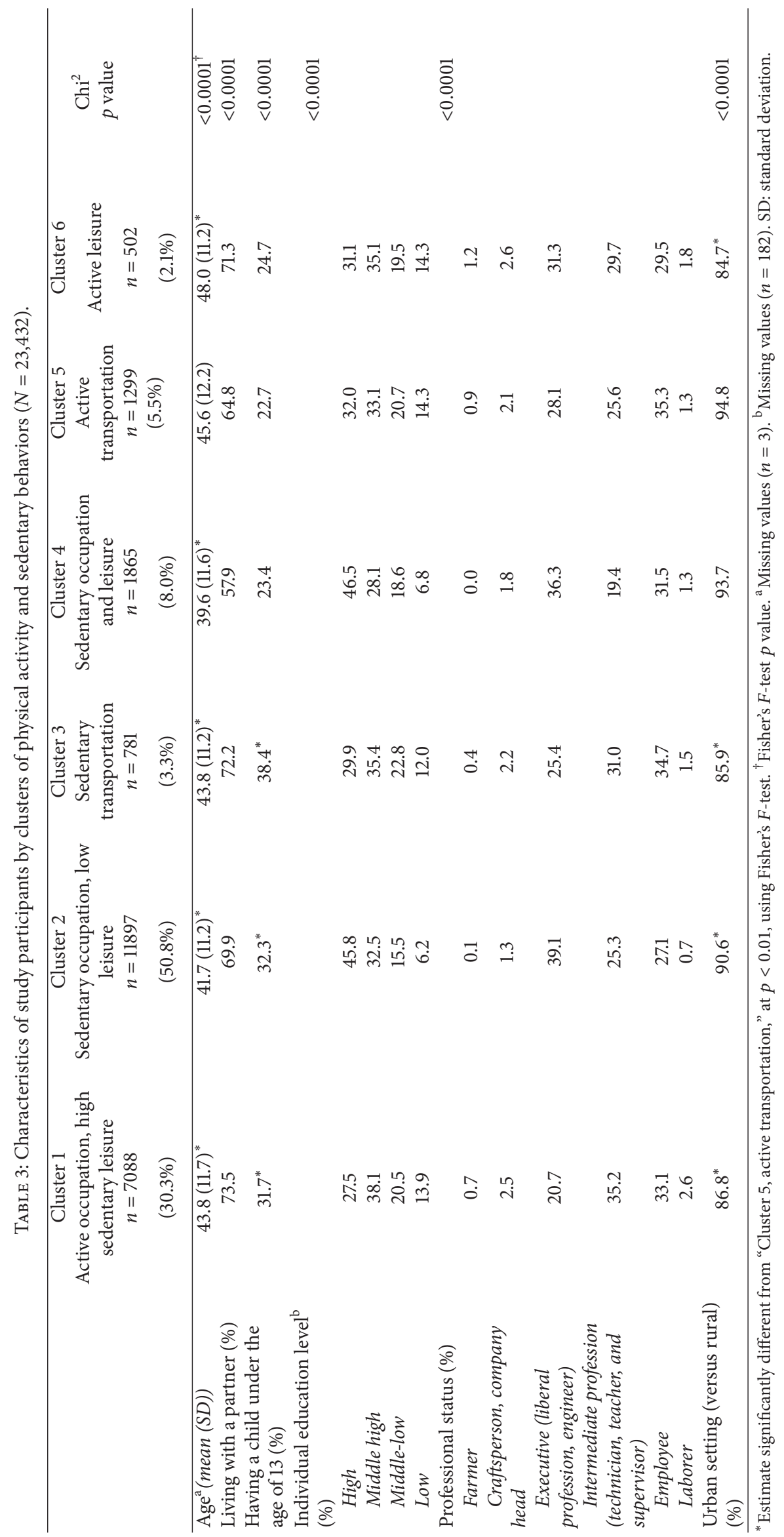




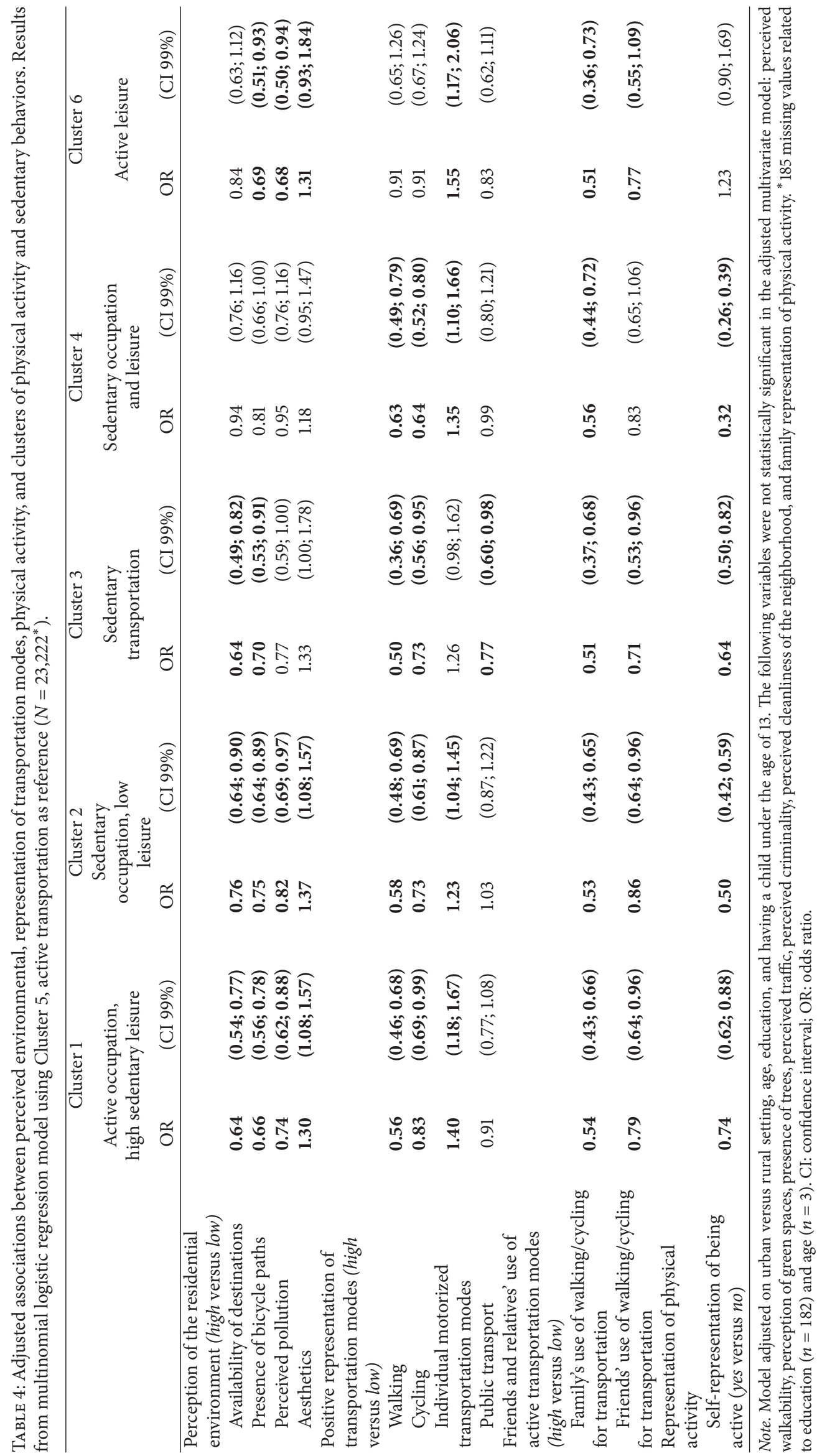


this latter, representing oneself as an active person decreased by $50 \%$ the odds of belonging to Cluster 2 .

3.3.3. Cluster 3 "Sedentary Transportation" versus Cluster 5 "Active Transportation". Perceiving a high availability of destinations and bicycle paths, having a high positive representation of walking, cycling, and using public transports, having relatives using active transportation modes, and representing oneself as an active person were associated with a decrease in the odds of being in Cluster 3 "sedentary transportation" compared with Cluster 5.

\subsubsection{Cluster 4 "Sedentary Occupation and Leisure" versus} Cluster 5 "Active Transportation". No perceived residential environment variables were correlated to the probability of belonging to Cluster 4 compared with Cluster 5 . Cluster 4 differed from Cluster 5 in representation of transportation modes, family's use of active transportation modes, and selfrepresentation of being an active person.

3.3.5. Cluster 6 "Active Leisure" versus Cluster 5 "Active Transportation". A high presence of bicycle paths and having friends and family members regularly using active transportation modes were associated with a strong decrease in the odds of being in Cluster 6 compared with Cluster 5. In contrast, living in a perceived low pollution and highly aesthetic neighborhood and having a positive representation of individual motorized transportation modes were associated with an increase in the odds of being in Cluster 6.

\section{Discussion}

In this study of a large sample of French working women, cluster analyses of detailed context-specific PA and SB data revealed an original typology of 6 distinct movement behavior patterns in daily life contexts. A specific cluster characterizing individuals with high levels of active transportation was identified as a unique pattern regarding individual and environmental correlates.

The present analysis highlights the variety of existing combinations of PA and SB according to contexts such as work, transportation, or leisure. Context-specific physical or sedentary activities have been related to distinct health effects $[16,27,28]$ and their cooccurrence could have a synergistic or antagonistic impact on health risk profile. This reinforces the importance of comprehensive analyses of daily life activities patterns and their correlates, to identify at-risk behavioral profiles for chronic diseases and mortality $[5,29]$.

As previously reported $[17,18,20]$, we observed that activities are not exclusive nor simply in opposition but rather cooccur throughout the day. For instance, the "sedentary transportation" cluster presents the second highest time spent in active transportation. This mixed pattern has also been observed in previous works $[30,31]$, as those who report high overall transportation time may have more opportunity of mixing passive and active means. The "active leisure" cluster and "active transportation" cluster presented the highest levels of reported PA (excluding occupational), consistent with previous reports from both US and French cohorts [17, 18].
Cross-sectional and longitudinal studies that investigated the association between leisure and transportation physical activities suggested that higher levels of active transportation are related to higher level of overall and leisure PA and not to a substitution of one context-related activity by another $[6,14,32]$. In our study, clusters with the lowest active transportation time also displayed the lowest time spent active in total or during leisure, but not necessarily the highest time spent sedentary. This confirms that interventions to promote active transportation should take into account the overall movement-related behavior of individuals, targeting both sedentary and active components accordingly.

In this sense, it is well recognized that context-specific PA $[9,33,34]$ and SB $[35,36]$ relate to distinct factors. Nevertheless, only few studies have simultaneously addressed the different components of movement behavior, by either focusing on one component and adjusting for another or by separately examining patterns of SB et PA $[16,17,20$, $29,37]$. Presently, in order to analyze active transportation behavior, we have specifically used cluster analysis to capture integrative patterns and to investigate the correlates of active transportation behavior within global movement activities rather than as an isolated behavior.

An important finding in this study is that the correlates of one of the clusters, termed "active transportation," were clearly distinct from those of all the other 5 clusters. This was evidenced through a detailed investigation of individual, social, and environmental factors that could differentiate the "active transportation" cluster from the other patterns of movement. The perceived environmental characteristics positively associated with the probability of belonging to the cluster "active transportation" as opposed to other clusters include high availability of destinations around the home, the presence of bicycle paths, and low traffic. Unexpectedly, the perception of a polluted residential area and a perceived low aesthetic environment were also positively associated with the cluster "active transportation." Such inverse associations have been observed elsewhere and may reflect that highly walk/bike-friendly environments are likely to be located in urban areas concentrating high multimodal transportation facilities and thus highly traffic polluted $[38,39]$. As previously documented, active transportation has been associated with environment features [40], such as higher residential density, mixed land use, street connectivity, access to destinations, and walking/cycling facilities [40, 41], with mixed results regarding aesthetics [11, 12] or safety [40, 42-44]. Previous literature also suggests that physical environmental attributes may be more related to active transportation than active leisure [45]. Concomitantly, environmental factors were strong correlates of belonging to the "active leisure" cluster compared to the "active transportation" cluster. Our results confirm the crucial link between perceived environment and active transportation pattern.

Regarding social factors, a high use of active transportation modes by relatives and friends was significantly associated with all patterns of movements compared to the "active transportation" cluster, this being even stronger for a family's use. When reviewing the social determinants of active travel in adults, Panter and Jones reported that active commuting 
was associated with social support from family and friends in Europe, with less consistency in Australia or the US [31]. In Europe, relatives' level of PA, walking/cycling with siblings or friends, was positively associated with total active transportation as well as social norms, social modelling, and social support [46-48].

We also investigated the link between the representation of different transportation modes and PA on patterns of movement. A positive representation of walking/cycling, a negative representation of individual motorized transportation, and the individual feeling of being physically active were positively associated with the probability of belonging to the cluster "active transportation." Longitudinal findings from the UK reported that alternatives to car use could be predicted by a lower favorable attitude towards the car [30].

In our study, compared to the referent cluster, the "sedentary transportation" cluster was not associated with representation of individual motorized transport modes but was negatively associated with a positive representation of public transport. Of note, this cluster displayed the highest time spent for transportation and second highest time for active transportation. This suggests that sedentary and active transportation behaviors are not exclusive or opposed and can cooccur more particularly in individuals reporting high transportation time.

Overall, the directions of the associations with the different studied factors were mainly similar for all clusters compared to the "active transportation" cluster, although we observed some slight variations in the significance and the strength of these associations from one cluster to another.

\section{Strengths and Limitations}

The originality of this study lies in the socioecological approach to the active transportation pattern's correlates as an integrated combination of daily life activities. One other strength is the large sample size and the specific validated questionnaires on the time spent in PA and SB in daily life contexts [24], with a detailed assessment of the perception of environment and transportation modes.

Nevertheless, our study presents some limitations. Its cross-sectional design prevents drawing any causal relationships. Self-reported questionnaires rather than objective measures were used to assess both context-specific PA and SB which could be a source of potential biases. It has been evidenced that sedentary SB can displace physical activity time, both moderate to vigorous (MVPA) and light intensity (LPA) [20, 29]. Physical activities encompass MVPA and LPA that also are interdependent in terms of time allocated and health effects $[29,37,49]$. Presently we did not subdivide LPA and MVPA. The workplace or leisure environments were not taken into account resulting in potential environment misrepresentation [50]. The analysis was performed on a female sample, included on a voluntary basis with relatively more highly educated individuals compared to the National Census data and with a large majority residing in urban areas [51], limiting extrapolation of the results to other populations. Preferences regarding residential location were not assessed, precluding residential neighborhood self-selection to be taken into account [52].

\section{Conclusion}

The identification of patterns of context-specific PA and SB is a step forward in the understanding of the complexity of movement behaviors towards a refined approach of active transportation and related correlates. Targeting patternspecific correlates will help to design dedicated intervention programs for active transportation and physical activity in specific subgroups.

\section{Conflicts of Interest}

The authors declare that there are no conflicts of interest regarding the publication of this paper.

\section{Acknowledgments}

The authors thank Younes Esseddik, Paul Flanzy, Mohand Ait Oufella, Yasmina Chelghoum, and Than Duong Van (computer scientists), Florence Charpentier (dietitian), Elise Martin (communication assistant), Nathalie Arnault, Véronique Gourlet, Fabien Szabo, Laurent Bourhis, and Stephen Besseau (statisticians), and Rachida Mehroug (logistics assistant) for their technical contribution to the NutriNet-Santé study. This work is part of the ACTI-Cités project (coordinator: J. M. Oppert) carried out with financial support from the French National Cancer Institute (Institut National du Cancer, INCa) through the Social Sciences and Humanities and Public Health Programme (2011-1-PL-SHS-10). The NutriNet-Santé cohort study is funded by the following public institutions: Ministère de la Santé, Institut de Veille Sanitaire (InVS), Institut National de la Prévention et de l'Education pour la Santé (INPES), Fondation pour la Recherche Médicale (FRM), Institut National de la Santé et de la Recherche Médicale (INSERM), Institut National de la Recherche Agronomique (INRA), Conservatoire National des Arts et Métiers (CNAM), and Université Paris 13. Menai Mehdi was funded by a grant from Fondation Le Roch-Les Mousquetaires.

\section{References}

[1] D. E. R. Warburton, C. W. Nicol, and S. S. D. Bredin, "Health benefits of physical activity: the evidence," Canadian Medical Association Journal, vol. 174, no. 6, pp. 801-809, 2006.

[2] M. Reiner, C. Niermann, D. Jekauc, and A. Woll, "Long-term health benefits of physical activity-a systematic review of longitudinal studies," BMC Public Health, vol. 13, no. 1, article 813, pp. 1-9, 2013.

[3] J. F. Sallis, F. Bull, R. Guthold et al., "Progress in physical activity over the Olympic quadrennium," The Lancet, vol. 388, no. 10051, pp. 1325-1336, 2016.

[4] A. Biswas, P. I. Oh, G. E. Faulkner et al., "Sedentary time and its association with risk for disease incidence, mortality, and hospitalization in adults: a systematic review and metaanalysis," Annals of Internal Medicine, vol. 162, no. 2, pp. 123-132, 2015. 
[5] U. Ekelund, J. Steene-Johannessen, W. J. Brown et al., "Does physical activity attenuate, or even eliminate, the detrimental association of sitting time with mortality? A harmonised metaanalysis of data from more than 1 million men and women," The Lancet, vol. 388, no. 10051, pp. 1302-1310, 2016.

[6] B. Chaix, Y. Kestens, S. Duncan et al., "Active transportation and public transportation use to achieve physical activity recommendations? A combined GPS, accelerometer, and mobility survey study," International Journal of Behavioral Nutrition and Physical Activity, vol. 11, no. 1, article no. 124, 2014.

[7] L. Foley, J. Panter, E. Heinen, R. Prins, and D. Ogilvie, "Changes in active commuting and changes in physical activity in adults: A cohort study," International Journal of Behavioral Nutrition and Physical Activity, vol. 12, no. 1, article no. 161, 2015.

[8] P. Gordon-Larsen, M. C. Nelson, and K. Beam, "Associations among active transportation, physical activity, and weight status in young adults," Obesity Research, vol. 13, no. 5, pp. 868-875, 2005.

[9] A. E. Bauman, R. S. Reis, J. F. Sallis, J. C. Wells, R. J. F. Loos, and B. W. Martin, "Correlates of physical activity: why are some people physically active and others not?" The Lancet, vol. 380, no. 9838, pp. 258-271, 2012.

[10] M. Menai, H. Charreire, T. Feuillet et al., "Walking and cycling for commuting, leisure and errands: Relations with individual characteristics and leisure-time physical activity in a crosssectional survey (the ACTI-Cités project)," International Journal of Behavioral Nutrition and Physical Activity, vol. 12, no. 1, article no. 150, 2015.

[11] B. E. Saelens, J. F. Sallis, and L. D. Frank, "Environmental correlates of walking and cycling: findings from the transportation, urban design, and planning literatures," Annals of Behavioral Medicine, vol. 25, no. 2, pp. 80-91, 2003.

[12] Y. Liao, I.-T. Wang, H.-H. Hsu, and S.-H. Chang, "Perceived environmental and personal factors associated with walking and cycling for transportation in Taiwanese adults," International Journal of Environmental Research and Public Health, vol. 12, no. 2, pp. 2105-2119, 2015.

[13] D. Van Dyck, E. Cerin, T. L. Conway et al., "Perceived neighborhood environmental attributes associated with adults' transport-related walking and cycling: Findings from the USA, Australia and Belgium," International Journal of Behavioral Nutrition and Physical Activity, vol. 9, article no. 70, 2012.

[14] S. Titze, W. J. Stronegger, S. Janschitz, and P. Oja, "Association of built-environment, social-environment and personal factors with bicycling as a mode of transportation among Austrian city dwellers," Preventive Medicine, vol. 47, no. 3, pp. 252-259, 2008.

[15] B. Giles-Corti and R. J. Donovan, "Relative influences of individual, social environmental, and physical environmental correlates of walking," The American Journal of Public Health, vol. 93, no. 9, pp. 1583-1589, 2003.

[16] H. Charreire, R. Casey, P. Salze et al., "Leisure-time physical activity and sedentary behavior clusters and their associations with overweight in middle-aged French adults," International Journal of Obesity, vol. 34, no. 8, pp. 1293-1301, 2010.

[17] A. Y. Omorou, J. Coste, H. Escalon, and A. Vuillemin, "Patterns of physical activity and sedentary behaviour in the general population in France: Cluster analysis with personal and socioeconomic correlates," Journal of Public Health (United Kingdom), vol. 38, no. 3, pp. 483-492, 2016.

[18] L. S. Rovniak, J. F. Sallis, B. E. Saelens et al., "Adults' Physical Activity Patterns Across Life Domains: Cluster Analysis With Replication," Health Psychology, vol. 29, no. 5, pp. 496-505, 2010.
[19] K. R. Evenson, F. Wen, J. S. Metzger, and A. H. Herring, "Physical activity and sedentary behavior patterns using accelerometry from a national sample of United States adults," International Journal of Behavioral Nutrition and Physical Activity, vol. 12, no. 1, article 20, 2015.

[20] S. Zwolinsky, J. McKenna, A. Pringle et al., "Physical activity and sedentary behavior clustering: segmentation to optimize active lifestyles," Journal of Physical Activity and Health, vol. 13, no. 9, pp. 921-928, 2016.

[21] M. Saidj, M. Menai, H. Charreire et al., "Descriptive study of sedentary behaviours in 35,444 French working adults: Crosssectional findings from the ACTI-Cités study," BMC Public Health, vol. 15, no. 1, article no. 379, 2015.

[22] S. Hercberg, K. Castetbon, S. Czernichow et al., "The nutrinetsanté study: A web-based prospective study on the relationship between nutrition and health and determinants of dietary patterns and nutritional status," BMC Public Health, vol. 10, article no. 242, 2010.

[23] T. Feuillet, P. Salze, H. Charreire et al., "Built environment in local relation with walking: Why here and not there?" Journal of Transport and Health, vol. 3, no. 4, pp. 300-312, 2016.

[24] K. Mensah, A. Maire, J.-M. Oppert et al., "Assessment of sedentary behaviors and transport-related activities by questionnaire: A validation study," BMC Public Health, vol. 16, no. 1, article no. 753, 2016.

[25] F. Hess, P. Salze, C. Weber, T. Feuillet, H. Charreire, M. Menai et al., "Active mobility and environment: a pilot qualitative study for the design of a new questionnaire," PLOS ONE, vol. 12, no. 1, 2017.

[26] B. Tabachnick and L. Fidell, Using Multivariate Statistics, 3rd ednHarper Collins, New York, NY, USA, 1996.

[27] G. Samitz, M. Egger, and M. Zwahlen, "Domains of physical activity and all-cause mortality: systematic review and doseresponse meta-analysis of cohort studies," International Journal of Epidemiology, vol. 40, no. 5, pp. 1382-1400, 2011.

[28] E. Stamatakis, J. Y. Chau, Z. Pedisic et al., "Are sitting occupations associated with increased all-cause, cancer, and cardiovascular disease mortality risk? A pooled analysis of seven british population cohorts," PLoS ONE, vol. 8, no. 9, Article ID e73753, 2013.

[29] S. F. M. Chastin, J. Palarea-Albaladejo, M. L. Dontje, D. A. Skelton, and J. Devaney, "Combined effects of time spent in physical activity, sedentary behaviors and sleep on obesity and cardio-metabolic health markers: a novel compositional data analysis approach," PLOS ONE, vol. 10, no. 10, Article ID e0139984, 2015.

[30] J. Panter, S. Griffin, A. M. Dalton, and D. Ogilvie, "Patterns and predictors of changes in active commuting over 12 months," Preventive Medicine, vol. 57, no. 6, pp. 776-784, 2013.

[31] J. R. Panter and A. Jones, "Attitudes and the environment as determinants of active travel in adults: What do and don't we know?" Journal of Physical Activity and Health, vol. 7, no. 4, pp. 551-561, 2010.

[32] S. Sahlqvist, A. Goodman, A. R. Cooper, and D. Ogilvie, "Change in active travel and changes in recreational and total physical activity in adults: longitudinal findings from the iConnect study," International Journal of Behavioral Nutrition and Physical Activity, vol. 10, article 28, 2013.

[33] J. F. Sallis, M. F. Floyd, D. A. Rodríguez, and B. E. Saelens, "Role of built environments in physical activity, obesity, and cardiovascular disease," Circulation, vol. 125, no. 5, pp. 729-737, 2012. 
[34] S. G. Trost, N. Owen, A. E. Bauman, J. F. Sallis, and W. Brown, "Correlates of adults' participation in physical activity: review and update," Medicine \& Science in Sports and Exercise, vol. 34, no. 12, pp. 1996-2001, 2002.

[35] R. E. Rhodes, R. S. Mark, and C. P. Temmel, "Adult sedentary behavior: A systematic review," American Journal of Preventive Medicine, vol. 42, no. 3, pp. e3-e28, 2012.

[36] G. O’Donoghue, C. Perchoux, K. Mensah et al., “A systematic review of correlates of sedentary behaviour in adults aged 18-65 years: A socio-ecological approach," BMC Public Health, vol. 16, no. 1, article no. 163, 2016.

[37] Ž. Pedišić, "Measurement issues and poor adjustments for physical activity and sleep undermine sedentary behaviour research-The focus should shift to the balance between sleep, sedentary behaviour, standing and activity," Kinesiology, vol. 46, no. 1, pp. 135-146, 2014.

[38] T. Feuillet, H. Charreire, M. Menai et al., "Spatial heterogeneity of the relationships between environmental characteristics and active commuting: Towards a locally varying social ecological model," International Journal of Health Geographics, vol. 14, no. 1, article no. 12, 2015.

[39] L. Mertens, J. Van Cauwenberg, A. Ghekiere et al., "Differences in environmental preferences towards cycling for transport among adults: A latent class analysis," BMC Public Health, vol. 16, no. 1, article no. 782, 2016.

[40] V. Van Holle, B. Deforche, J. Van Cauwenberg, L. Goubert, L. Maes, N. Van de Weghe et al., "Relationship between the physical environment and different domains of physical activity in European adults: a systematic review," BMC Public Health, vol. 12, no. 1, article 807, 2012.

[41] J. F. Sallis, T. L. Conway, L. I. Dillon et al., "Environmental and demographic correlates of bicycling," Preventive Medicine, vol. 57, no. 5, pp. 456-460, 2013.

[42] Z. Kerr, K. R. Evenson, K. Moore, R. Block, and A. V. Diez Roux, "Changes in walking associated with perceived neighborhood safety and police-recorded crime: The multi-ethnic study of atherosclerosis," Preventive Medicine, vol. 73, pp. 88-93, 2015.

[43] S. Foster, P. Hooper, M. Knuiman, H. Christian, F. Bull, and B. Giles-Corti, "Safe RESIDential Environments? A longitudinal analysis of the influence of crime-related safety on walking," International Journal of Behavioral Nutrition and Physical Activity, vol. 13, no. 1, article no. 22, 2016.

[44] B. De Geus, I. De Bourdeaudhuij, C. Jannes, and R. Meeusen, "Psychosocial and environmental factors associated with cycling for transport among a working population," Health Education Research, vol. 23, no. 4, pp. 697-708, 2008.

[45] C. M. Hoehner, L. K. Brennan Ramirez, M. B. Elliott, S. L. Handy, and R. C. Brownson, "Perceived and objective environmental measures and physical activity among urban adults," The American Journal of Preventive Medicine, vol. 28, no. 2, pp. 105116, 2005.

[46] I. De Bourdeaudhuij, P. J. Teixeira, G. Cardon, and B. Deforche, "Environmental and psychosocial correlates of physical activity in Portuguese and Belgian adults," Public Health Nutrition, vol. 8, no. 7, pp. 886-895, 2005.

[47] B. Deforche, D. Van Dyck, M. Verloigne, and I. De Bourdeaudhuij, "Perceived social and physical environmental correlates of physical activity in older adolescents and the moderating effect of self-efficacy," Preventive Medicine, vol. 50, pp. S24-S29, 2010.

[48] H. Verhoeven, D. Simons, D. Van Dyck et al., "Psychosocial and Environmental Correlates of Walking, Cycling, Public
Transport and Passive Transport to Various Destinations in Flemish Older Adolescents," PLoS ONE, vol. 11, no. 1, Article ID e0147128, 2016.

[49] G. N. Healy, E. A. Winkler, C. L. Brakenridge, M. M. Reeves, E. G. Eakin, and M. Alemany, "Accelerometer-derived sedentary and physical activity time in overweight/obese adults with type 2 diabetes: cross-sectional associations with cardiometabolic biomarkers," PLOS ONE, vol. 10, no. 3, Article ID e0119140, 2015.

[50] C. Perchoux, B. Chaix, S. Cummins, and Y. Kestens, "Conceptualization and measurement of environmental exposure in epidemiology: accounting for activity space related to daily mobility," Health and Place, vol. 21, pp. 86-93, 2013.

[51] V. A. Andreeva, B. Salanave, K. Castetbon et al., "Comparison of the sociodemographic characteristics of the large NutriNetSanté e-cohort with French Census data: The issue of volunteer bias revisited," Journal of Epidemiology and Community Health, vol. 69, no. 9, pp. 893-898, 2015.

[52] G. R. McCormack and A. Shiell, "In search of causality: a systematic review of the relationship between the built environment and physical activity among adults," International Journal of Behavioral Nutrition and Physical Activity, vol. 8, article 125, 2011. 


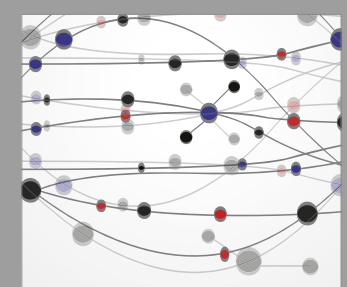

The Scientific World Journal
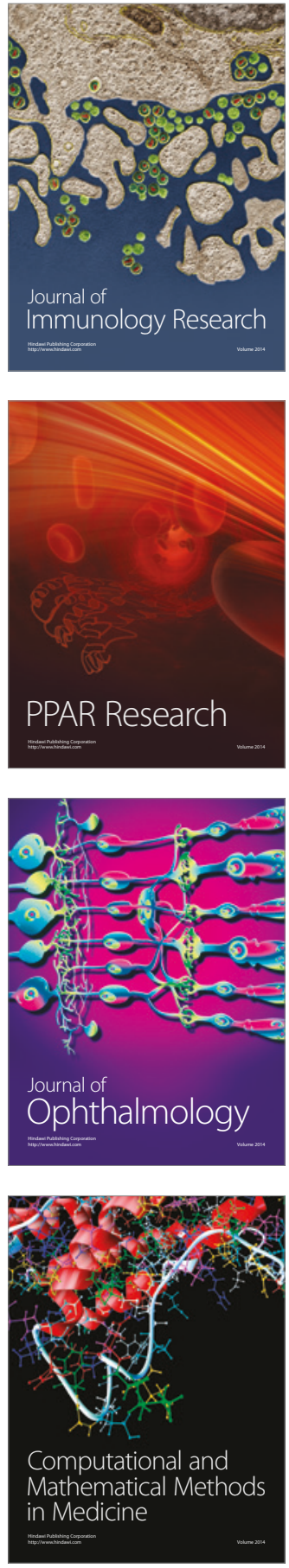

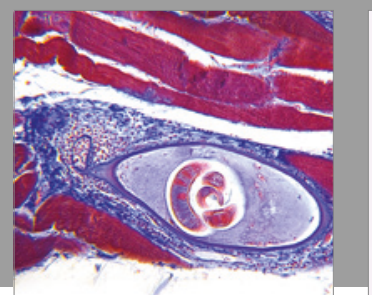

Gastroenterology Research and Practice
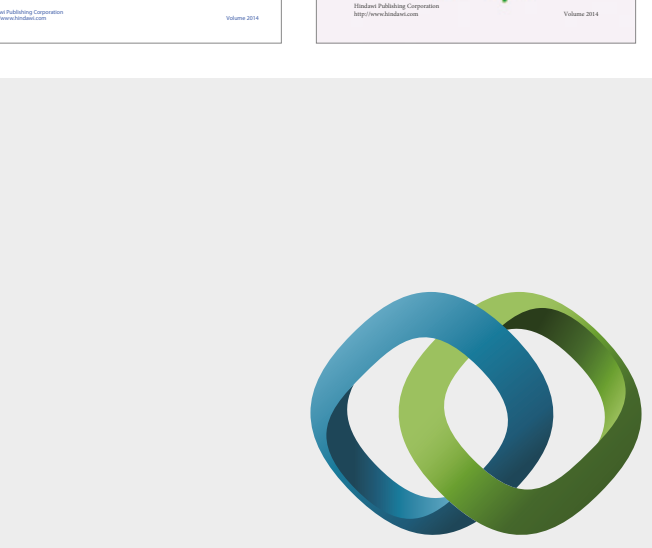

\section{Hindawi}

Submit your manuscripts at

https://www.hindawi.com
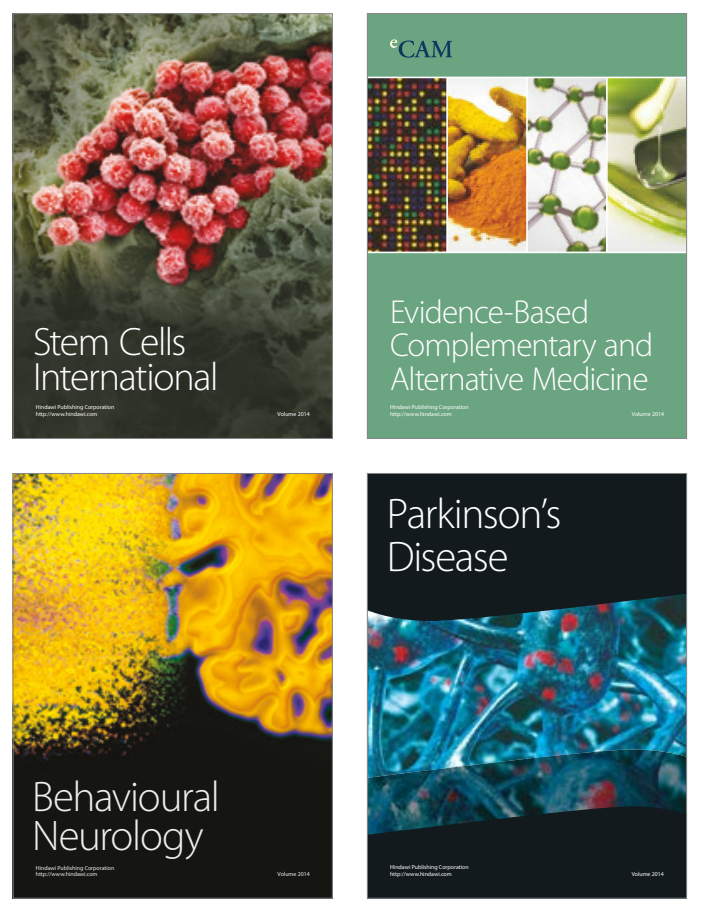
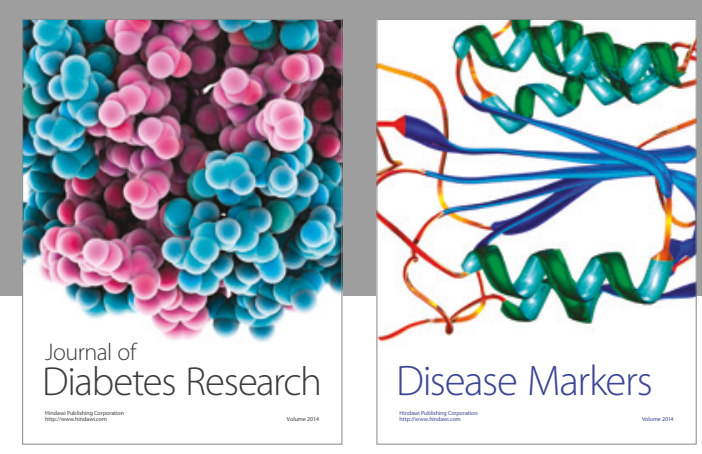

Disease Markers
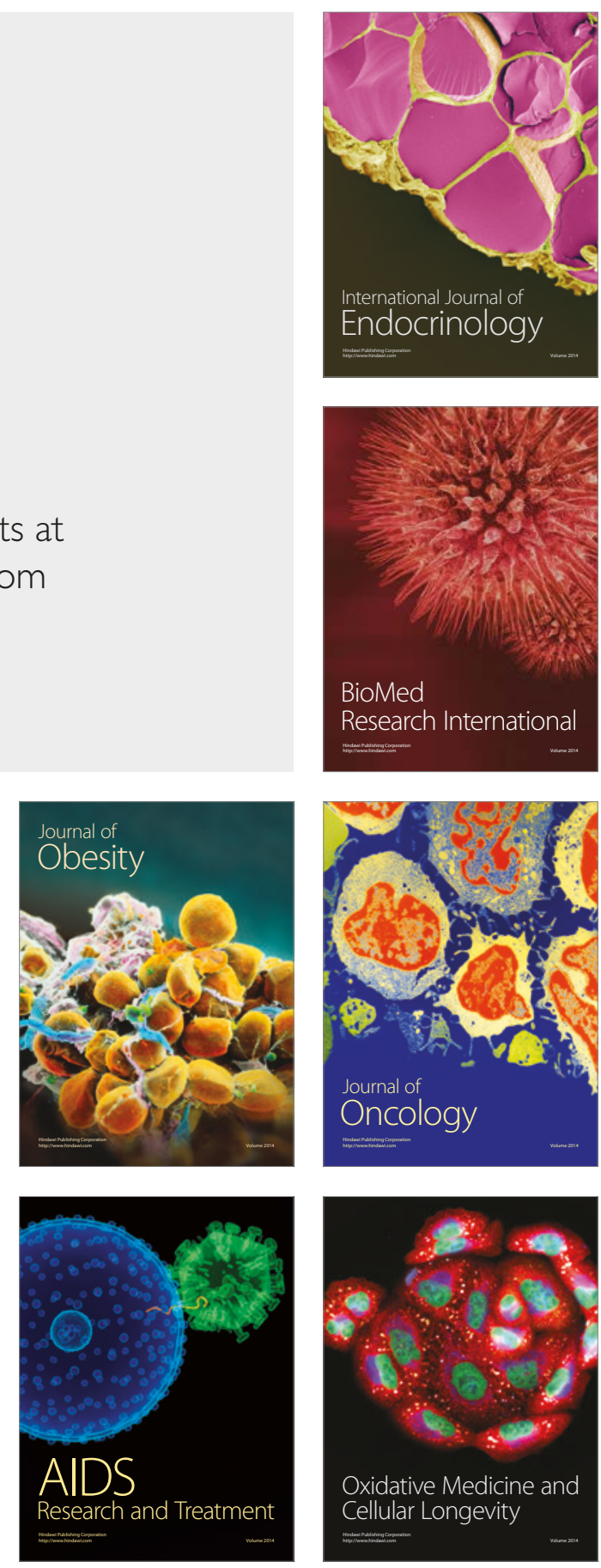